“C 2011 IEEE. Personal use of this material is permitted. Permission from IEEE must be obtained for all other uses, in any current or future media, including reprinting/republishing this material for advertising or promotional purposes, creating new collective works, for resale or redistribution to servers or lists, or reuse of any copyrighted component of this work in other works." 


\section{Review On Automatic Early Skin Cancer Detection}

\author{
Azadeh Noori Hoshyar \\ Department of Computer Science, \\ Faculty of Information Science and Technology, \\ University Kebangsaan Malaysia, \\ 43600 UKM Bangi,Malaysia \\ a_noori_h@yahoo.com
}

\author{
Adel Al- Jumaily \\ Faculty of Engineering and IT, \\ University of Technology, Sydney \\ Adel.Al-Jumaily@eng.uts.edu.au
}

Abstract- Skin cancer is increasing in different countries especially in Australia. Early detection of skin cancer can treat melanoma successfully, therefore, curability and survival depends directly on removing melanoma in its early stages. Since clinical observations face to different fault for melanoma detection, the automatic diagnosis can help to increase the accuracy of detection. Reviewing the research have done in skin cancer detection is the ultimate aim of this paper and to provide an overview on automatic detection of skin cancer. This paper presents the literature on automatic skin cancer detection and also describes the different steps of such process based on the implantation capabilities for such research.

Keywords-skin cancer detection; cancer classification; cancer segmentation; cancer feature extraction;automatic detection

\section{INTRODUCTION}

Malignant melanoma is nowadays one of the leading cancer have been increased in the last decades in Australia, America and Europe [1]. Fortunately, if the skin cancer is detected early, the curability is very high and over 92\% [2]. In many cases, dermatologists must perform a biopsy (a laboratory medical procedure) to determine whether a tumor is malignant or benign. Since this procedure involves some expense and morbidity, automatic early detection techniques are being as rapid and convenient skin cancer screening [3].

Dermatology imaging researchers believe that diagnosis of skin melanoma can be automated based on certain physical features and color information that are characteristic of the different categories of skin cancer [4]. It has been revealed that the major diagnostic and prognostic parameters of melanoma are the vertical thickness, three-dimensional (3D) size and shape, and color of the lesion. The other characteristic features of early melanoma includes irregularities in the boundary of the lesion, and the appearance of non-uniform pigmentation with a variety of color [4][5].

Many experimental researches attempt to build automatic skin cancer detection and improve the accuracy of diagnosis. In the following, the literatures on these attempts are reviewed. Also, in order to achieve a reliable skin cancer detection system, the right path knowledge which is explained in this paper seems crucial.

\section{LITERATURE REVIEW}

According to the importance of early detection, several companies and researchers have dedicated time and efforts to improve the early screening process. One of the most effective solutions already developed was the result of Australian Commonwealth Scientific and Industrial Research Organization research. It captures and analyses calibrated images of pigmented skin lesions that are stored for subsequent lesion monitoring or confirmation. The system was tested and one paper published in the application of the system [6]. In 1995, some researchers have been done on the application of Neural Classifiers to skin lesions classification purposes [7]. Also, on the subject of skin lesions differentiation, a paper published to identify the skin lesions using "skin line direction" [8]. In 2002, Bayes rule as one of the skin lesions classification method was published with rather inconclusive results [9]. Common classification methods like statistical and rule-based ones were applied in the researches of [10-12]. More advanced techniques such as Neural Network were presented in the works like [13-16]. Knearest neighborhood as another classification method was employed in the research of [17]. Furthermore, Classification and regression trees (CART) analysis has been applied in [18]. Finally, the ADWAT method for lesion classification was applied in [19] and [20].

\section{AUTOMATION OF SKIN CANCER DETECTION}

Automation of skin cancer detection can reduce the false positive or false negative clinical diagnosis because it adds a quantitative observation to the "clinical eye observation". The common approach to skin lesion early detection is divided into four stages of pre-processing, segmentation, feature extraction, and classification as figure1 $[21,22]$. 


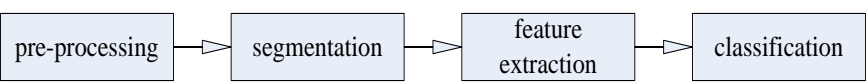

Figure 1.Common steps for skin lesion early detection

\section{A. Preprocessing}

Pre-processing is to perform image processing on original image to obtain the enhanced image. The common operations in pre-processing step are as follows; to reduce the processing time, images are resized to lower resolution pixels. To remove this bright area around the lesion a binary mask is generated and a new image is produced. This image is cropped for removing extra areas. In the next step, by performing some filtering the hairs are removed. Finally, to suppress large variations within the lesion and background, and also to reduce the effect of different skin color variations, the original color RGB images are transformed into intensity (grayscale) ones [23]. Figure 2 shows the sample of original and preprocessed image.

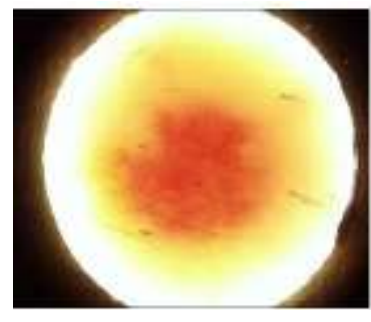

a

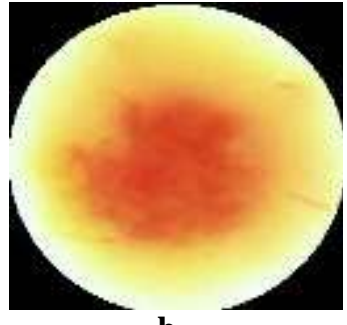

b
Figure 2. Sample of (a) original and (b) preprocessed image [23]

\section{B. Segmentation}

Segmentation is one of the important steps in cancer automatic detection, because it can greatly affect on the results of detection. An accurate segmentation of skin images can help the diagnosis to define well the region of the cancer. To obtain the information on lesion area, it is important to run a segmentation process to extract the pixels belonging to the lesion on skin image [24]. Therefore, in this step, the lesion is segmented from its surrounding skin. There are many techniques for segmentation based on different methods. There are four broad classes of segmentation methods, which are: classification-based methods, edgebased methods, region-based methods and, hybrid methods [25]. Figure 2 shows the sample of lesion segmentation after preprocessing the image.
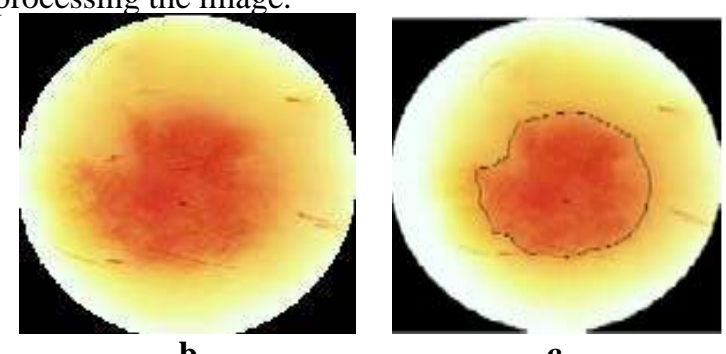

b

Figure 2. Sample of (b) preprocessed image (c) segmented image

\section{Feature extraction}

In this stage, the features of segmented lesion are extracted to feed into classifiers. The features extracted must be measurable and of high sensitivity, i.e., high correlation of the feature with skin cancer and high probability of true positive response. In addition, the features should have high specificity, i.e., high probability of true negative response [26].

Based on the features inspected by dermoscopy, different diagnostic models have become more reliable by clinicians:

- Pattern analysis: The pattern analysis method try to identify specific patterns, which may be global (reticular, globular, cobblestone, homogeneous, starburst, parallel, multicomponent, nonspecific) or local (pigment network, dots/globules/moles, streaks, blue-whitish veil, regression structures, hypopigmentation, blotches, vascular structures) [26].

- The ABCD-rule of dermatoscopy: This method is based on a semi-quantitative assessment using four dermoscopic criteria: asymmetry (A), border (B), color (C) and different structures(D) [27].

- The ELM 7-point checklist: It is a scoring diagnosis analysis and considers only seven standard ELM criteria includes atypical pigment network, blue-whitish veil, atypical vascular pattern, irregular streaks, irregular dots/globules, irregular blotches, and regression structures[26][27].

- Menzies Method: This method seek negative features such as symmetry of pattern and presence of a single color, also positive features such as blue-white veil, multiple brown dots, pseudopods, radial streaming, scarlike depigmentation, peripheral black dots/globules, multiple (five to six) colors, multiple blue/gray dots and broadened network[26].

- Texture Analysis: This method try to quantify texture notions such as "fine," "rough," and "irregular" and to identify, measure, and utilize the differences between them[26].

These extracted parameters constitute the entry of the stage of classification.

\section{Classification}

The final process of automatic skin cancer detection is to classify the extracted features of lesions into either malignant or benign. At the classification stage, after a satisfactory classifier is built, it is used to classify any new instances coming in [28]. There are different classification models which can be grouped into general classes as follows [29];

- Global Models such as Linear Models, Polynomial Models, Neural Networks (MLP), Support Vector Machines 
- Semi-global Models such as Radial Basis Functions, Multivariate Adaptive Regression Splines (MARS), Decision Trees (C4.5, CART)

- Local Models such as k-Nearest-Neighbors

- Hybrid Models such as Projection Based Radial Basis Functions Network (PRBFN)

After building a suitable classifier for automatic skin cancer detection, it is identified whether the lesion is malignant. Classification is considered as a significant task in building and developing reliable automated systems for diagnosis of skin lesions [26].

\section{CONCLUSION}

As the importance of early detection of skin cancer in treatment, this paper reviewed a literature on automatic cancer detection, also defined required steps for automating skin cancer detection. In each step, the techniques and methods which are useful in the process were mentioned. The automated skin cancer system can be well designed as a substitute of clinician in melanoma diagnosis.

\section{REFERENCES}

[1] R. Marks, "Epidemiology of melanoma", Clin. Exp. Dermatology Vol. 25, pp. 459-463, 2000

[2] U. Leiter, P. G. Buttner et alii, "Prognostic factors of thin cutaneous melanoma: an analysis of the central malignant melanoma registry of the German Dermatological Society", Journal of Clinical Oncology, Vol. 22, pp. 3660-3667, 2004.

[3] Fikret Ercal, Anurag Chawla, William V. Stoecker, Hsi-Chieh Lee, and Randy H. Moss." Neural Network Diagnosis of Malignant Melanoma From Color Images", IEEE TRANSACTIONS ON BIOMEDICAL ENGINEERING. VOL. 41, NO. 9, SEYIEMBER 1994

[4] I.M. Ariel, "Malignant Melanoma", Appleton- Century-Crofts, New York, 1981.

[5] A.J. Sober, "Diagnosis and management of skin-cancer", Cancer, vol. 51, pp. 2448-2452, 1983.

[6] Menzies et al. - The Performance of SolarScan - Arch Dermatol. November 2005;141:1388-1396

[7] Hintz-Madsen, M., Hansen, L., Larsen, J., Olesen, E. and Drzewiecki, K. - Design and Evaluation of Neural Classifiers Application to Skin Lesion Classification - Proceedings of the 1995 IEEE Workshop on Neural Networks for Signal Processing

[8] She, Z. and Fish, P. - Skin Lesion Differentiation Using Skin Line Direction - School of Informatics, University of Wales

[9] Sigurdsson, S., Larsen, J., Hansen, L., Philipsen, P and Wulf, H. Outlier Estimation and Detection Application to Skin Lesion Classification - International conference on acoustics, speech and signal processing - Vol. 1 pp. 1049-1052 - 2002

[10] S.W. Menzies, L.Bischof,H. Talbot,A.Gutener,M.Avramidis, L.Wong et al., "The performance of SolarScan-An automated dermoscopy image analysis instrument for the diagnosis of primary melanoma," Arch. Dermatol., vol. 141, no. 11, pp. 1388-1396, 2009.

[11] A. Blum, H. Luedtke, U. Ellwanger, R. Schwabe, G. Rassner, and C. Garbe, "Digital image analysis for diagnosis of cutaneous melanoma. Development of a highly effective computer algorithm based on analysis of 837 melanocytic lesions," Br. J. Dermatol., vol. 151, no. 5, pp. 1029-1038, Nov. 2004.
[12] C. Grana, G. Pellacani, R. Cucchiara, and S. Seidenari, "A new algorithm for border description of polarized light surface microscopic images of pigmented skin lesions," IEEE Trans.Med. Imag., vol. 22, no. 8, pp. 959-964, Aug. 2003.

[13] J. Boldrick, C. Layton, J. Ngyuen, and S. Swtter, "Evaluation of digital dermoscopy in a pigmented lesion clinic: Clinician versus computer assessment of malignancy risk," J. Amer. Acad. Dermatol., vol. 56, no. 3, pp. 417-421, Mar. 2007.

[14] G. Surowka and K. Grzesiak-Kopec, "Different learning paradigms for the classification of melanoid skin lesions using wavelets," in Proc. 29th Annu. Int. Conf. IEEE EMBS, Aug. 2007, pp. 3136-3139.

[15] W. V. Stoecker, K. Gupta, R. J. Stanley, R. H. Moss, and B. Shrestha, "Detection of asymmetric blotches in dermoscopy images of malignant melanoma using relative color," Skin Res. Technol., vol. 11, no. 3, pp. 179-184, Aug. 2005.

[16] S. Sigurdsson, P. A. Philipsen, L. K. Hansen, J. Larsen, M Gniadecka, and H. C. Wulf, "Detection of skin cancer by classification of raman spectra," IEEE Trans. Biomed. Eng., vol. 10, no. 51, pp. 1784-1793, Oct. 2004.

[17] H. Ganster, P. Pinz, R. Rohrer, E. Wildling, M. Binder, and H. Kittler, "Automated melanoma recognition," IEEE Trans. Med. Imag., vol. 20, no. 3, pp. 233-239, Mar. 2001.

[18] M.Wiltgen, A. Gerger, and J. Smolle, "Tissue counter analysis of benign common nevi and malignant melanoma," Int. J. Med. Inf., vol. 69, pp. 17-28, 2003.

[19] S. V. Patwardhan, S. Dai, and A. P. Dhawan, "Multi-spectral image analysis and classification of melanoma using fuzzy membership based partitions," Comput. Med. Imag. Graph., vol. 29, pp. 287-296, 2005.

[20] S. V. Patwardhan, A. P. Dhawan, and P. A. Relue, "Classification of melanoma using tree structured wavelet transforms," Comput. Methods Programs Biomed., vol. 72, pp. 223-239, 2003.

[21] Andy Chiem, Adel Al-Jumaily, and Rami N. Khushaba," A Novel Hybrid System for Skin Lesion Detection”, IEEE, 2007

[22] Ho Tak Lau, Adel Al-Jumaily," Automatically Early Detection of Skin Cancer: Study Based on Nueral Netwok Classification", International Conference of Soft Computing and Pattern Recognition, 2009.

[23] G. Zouridakis, M. Doshi, M. Duvic, and N.A. Mullani," TRANSILLUMINATION IMAGING FOR EARLY SKIN CANCER DETECTION", Technical Report University of Houston,2005.

[24] O.Colt,R.Devinoy,A.Sombo,D.de Brucq," A Colour Image Processing Method for Melonoma Detection", first international conference, Cambridge, MA, USA, October 11-13, 1998 : proceedings

[25] Gonzalez, R.C. and R.E. Woods, 2008. Digital Image Processing. 3rd Edn., Prentice- all, Inc., New Jersey, ISBN: 10: 013168728x, pp: 594.

[26] Ilias Maglogiannis, Charalampos N. Doukas," Overview of Advanced Computer Vision Systems for Skin Lesions Characterization", IEEE TRANSACTIONS ON INFORMATION TECHNOLOGY IN BIOMEDICINE, VOL. 13, NO. 5, SEPTEMBER 2009

[27] G. Di Leo, A. Paolillo, P. Sommella, G. Fabbrocini, O. Rescigno," A software tool for the diagnosis of melanomas, Automatic Implementation of the 7-Point Check List Method", IEEE,2010

[28] Xiaojing Yuan, Zhenyu Yang, George Zouridakis and Nizar Mullani," SVM-based Texture Classification and Application to Early Melanoma Detection", Proceedings of the 28th IEEE EMBS Annual International Conference New York City, USA, Aug 30-Sept 3, 2006

[29] M. J. Ogorzałek, G. Surówka, L. Nowak, C. Merkwirth," New Approaches for Computer-Assisted Skin Cancer Diagnosis", The Third International Symposium on Optimization and Systems Biology (OSB’09) Zhangjiajie, China, September 20-22, 2009 